\title{
QUANTITATIVE DETERMINATION OF HEAVY METAL HYPERACCUMULATION IN A MACROPHYTE SAMPLE OF SCHOENOPLECTUS CALFORNICUS FROM LAGO SAN PABLO, IMBABURA-ECUADOR
}

\author{
Suly Rodríguez Ayala (1), Ronny Flores ${ }^{(2)}$, Monserrat Rodríguez ${ }^{(3)}$ y Marcelo \\ Andocilla $^{(4)}$ \\ ${ }^{(1)}$ Carrera de Ingeniería Ambiental. Universidad Central del Ecuador UCE. Av. América y Plaza \\ Indoamérica. Ciudadela Universitaria, Ecuador. smrodriguez@uce.edu.ec \\ ${ }^{(2)}$ Carrera de Química Pura. Universidad Central del Ecuador UCE. Quito, Ecuador \\ ${ }^{(3)}$ Carrera de Medicina. Universidad de la Américas UDLA. Quito, Ecuador \\ ${ }^{(4)}$ Carrera de Ciencias Biológicas. Universidad Central del Ecuador UCE. Quito, Ecuador
}

Received: December 2016. Accepted: July 2017

\begin{abstract}
The aim of this study is to quantify heavy metal hyperaccumulation such as nickel, arsenic, cadmium, mercury, lead and chromium in shoots and roots of the aquatic plant with phytoremediation ability known as Schoenoplectus californicus or totora, located in benthic zones of Araque Ravine, an affluent of Lake San Pablo in Imbabura-Ecuador. We used the instrumental method of atomic absorption spectroscopy in graphite furnace and hydride generator: the first one for the determination of concentrations of nickel, cadmium, chromium and lead, and the second one to determine the one of arsenic and mercury. This investigation found that significant levels of nickel, arsenic, cadmium and mercury were cuantified in the roots in contrast to the concentrations obtained of these heavy metals in shoots and no chromium and lead values were detected in this aquatic plant. Therefore, Schoenoplectus californicus is a hyperaccumulator macrophyte because the high accumulated concentrations of nickel, arsenic and cadmium were above the threshold of hyperaccumulation and this property could allow it to be used in the decontamination of soils and phytomining
\end{abstract}

Keywords: Inorganic contaminants, hydrophytes, totora, bioacumulation, rhizomes.

\section{DETERMINACIÓN CUANTITATIVA DE LA HIPERACUMULACIÒN DE METALES PRESENTES EN UNA MUESTRA DE MACRÓFITOS DE SCHOENOPLECTUS CALIFORNICUS DEL LAGO SAN PABLO, IMBABURA-ECUADOR}

\section{RESUMEN}

El objetivo del presente trabajo de investigación es la determinación cuantitativa de la hiperacumulación de los metales pesados como níquel, arsénico, cadmio, mercurio, plomo y cromo en los tallos y raíces de la planta acuática con características fitodepuradadoras conocida como Schoenoplectus californicus o 
totora, ubicada en las zonas bentónicas de la Vertiente Apangora del Lago San Pablo de Imbabura-Ecuador. Se utilizó el método instrumental de espectroscopia de absorción atómica en horno de grafito y generador de hidruros; el primero para la determinación de las concentraciones de níquel, cadmio, cromo y plomo, y el segundo para determinar las de arsénico y mercurio. Como resultado se ha encontrado que, en las raíces, existen mayores niveles de níquel, arsénico, cadmio y mercurio en comparación con las concentraciones obtenidas de estos metales pesados en los tallos, mientras que no se han detectado valores de cromo y plomo en esta planta acuática. Por lo tanto, Schoenoplectus californicus es una planta hiperacumuladora; las elevadas concentraciones acumuladas de níquel, arsénico y cadmio se encuentran por encima del umbral de hiperacumulación y esta propiedad podría permitir que sea empleada en la descontaminación de suelos y en la fitominería.

Palabras claves: contaminantes inorgánicos, hidrófitos, totora, bioacumulación, rizomas.

\section{INTRODUCCIÓN}

En Ecuador, al pie del volcán Imbabura con una altitud de aproximadamente 2.650 metros (Terneus, 2014), se encuentra la laguna San Pablo o Imbakucha (Cevallos, 2015c). Esta laguna descrita como como eutrófica (grandes cantidades de nutrientes) (Cevallos, 2015c), tiene una profundidad máxima de 30 metros (Cevallos, 2015c) con un agua transparente hasta los 3.5 metros (Terneus, 2014).

La formación de sus cuerpos de agua proviene del deshielo del volcán, las precipitaciones y las redes hídricas superficiales y subterráneas (Cevallos, 2015c); entre sus afluentes se encuentran los ríos Itambi (Casallas \& Gunkel, 2001) y Manantial (Terneus, 2014) y las quebradas Araque, Túqueres, Imbaburita y Macufishi (Cevallos, 2015c), mientras que su efluente es el denominado "Desaguadero" (Casallas \& Gunkel, 2001).

Además, su extensión de 615,6 hectáreas (Jurado, Jaramillo, \& Reyes, 2015) está rodeada por cuatro parroquias rurales del cantón Otavalo en la provincia de Imbabura (Ver Figura 1): San Pablo del Lago (SP), Eugenio Espejo (EE), González Suárez (GS) y San Rafael (SR) (Terneus, 2014); tienen una población total de 28.309 personas (SP: 9901, EE: 7357, GS: 5630 y SR: 5421) (Cevallos, 2011, 2015a, 2015b, 2015c). 


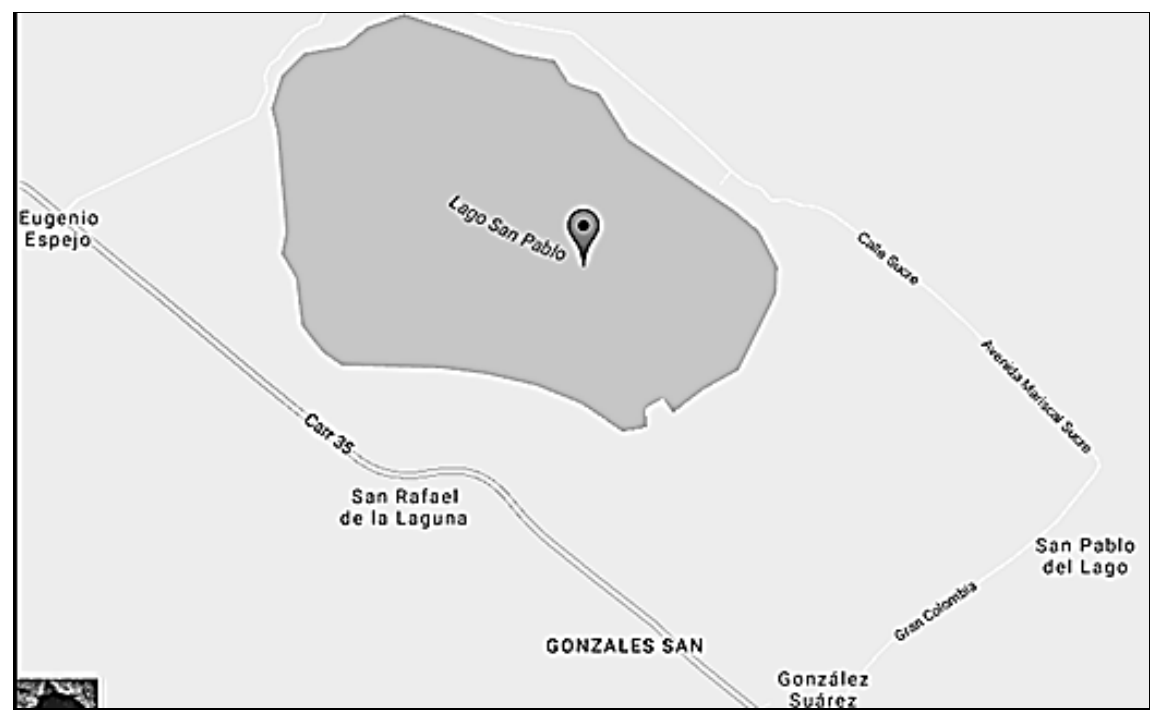

Figura 1 Parroquias rurales en el Lago San Pablo (Imbabura-Ecuador)

Según el informe del Sistema Nacional de Información de Ecuador, en Otavalo hay en un clima ecuatorial mesotérmico semi-húmedo (clasificación Pourrut) con una temperatura de entre 10 a $20 \circ \mathrm{C}$, humedad del 65 al $85 \%$ y precipitaciones de 1000 a 2000 mm (Sistema Nacional de Información, 2011) con dos pares de meses de precipitaciones máximas, octubre-noviembre y enero-abril (Instituto Nacional de Meteorología e Hidrología, 2012). Las parroquias aledañas al lago presentan un ecosistema altoandino húmedo en las que se desarrollan especies herbáceas no superiores a 1,5 metros (Jurado et al., 2015) entre las que se encuentran Schoenoplectus californicus, Chusquea sp., Typha latifolia, Bidens andicola, Eichhornia crassipes, Plantago australis Lam, Paspalium bonlandianum, Rorippa cf. clandestina, y Scirpus sp. (López, 2012) junto a pastizales y cultivos (Santander, Ágreda, \& Adriana, 2013). Últimamente se ha realizado un monitoreo de la fauna, determinándose por el momento 23 especies de aves acuáticas (Jurado et al., 2015) como pelícanos, garcetas, patos, garzas, garcetas, fochas, playeros, cigüeñuelas, gaviotas, patiamarillos y cormoranes (López, 2012), algunas de las cuales migran hasta la zona en algunas estaciones del año; en contraste, los peces encontrados en este lago incluyen la perca o lubina negra, la tilapia, los gruppis y los espada (Terneus, 2014).

Schoenoplectus californicus (C. A. Mey) o Scirpus californicus (Chester, Wofford, Estes, \& Bailey, 2009) con sus dos subespecies californicus y tatora (Heiser, 1978) son los nombres científicos de la especie a la que corresponden los juncos o las totoras (De Marco, Vega, \& Bellagamba, 2011), pertenecientes a la familia de las ciperáceas (Richardson \& King, 2010) y al orden Cyperales (USDA, n.d.). Los juncos o totoras son plantas perennes acuáticas con una altura de 1 a 3 metros (Richardson \& King, 2010), pudiendo alcanzar una profundidad de hasta 1 metro (Plan Ceibal, n.d.) y con rizomas dispuestos de forma horizontal (De Marco et al., 2011). La estructura de las totoras se divide en tres partes: el tallo, la inflorescencia y las raíces (Ninaquispe-Romero, Weeks, \& Huelman, 2012). 
En Ecuador, se ha encontrado evidencia de que solamente la subespecie californicus (como se detalla en la Figura 2), se encuentra en las tierras altas de la sierra (Heiser, 1978) entre las que se incluye el Lago San Pablo (Imbabura), Yaguarcocha (Imbabura), Cusín (Imbabura), Guaytacama (Cotopaxi), Tanicuchí (Cotopaxi), la Laguna de Colta (Chimborazo) y el Río Guano (Chimborazo) dentro de un área de 442 hectáreas (Macía \& Balslev, 2000), además de determinadas zonas costeras de Esmeraldas (Macía \& Balslev, 2000).

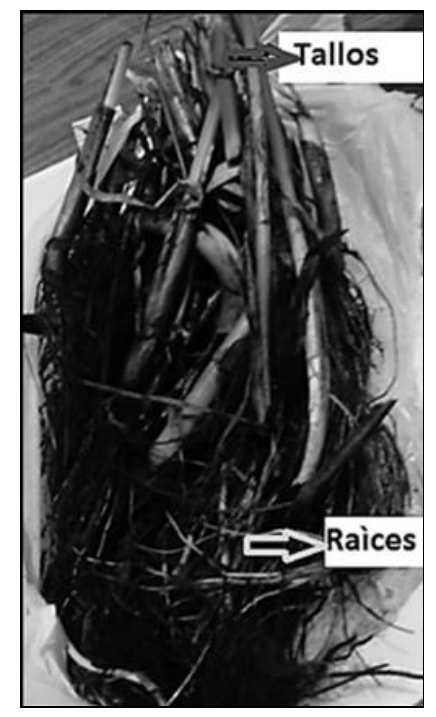

Figura 2 Schoenoplectus californicus. Lago San Pablo (Imbabura-Ecuador)

\section{Introducción al problema}

Este lago se ha convertido en un centro turístico importante para el cantón con actividades como concursos de vela y natación y paseos en lancha, además, se ha determinado que el sector de Punyaru es vulnerable a inundaciones como consecuencia del incremento del nivel del agua en el lago y ciertas parroquias del Lago San Pablo a los incendios forestales (Jurado et al., 2015). Así, existen informes que afirman que la actividad antropológica ha mermado la fauna y flora del lago por efecto de la cacería y navegación, el uso directo del agua, la contaminación con desechos domésticos y sustancias químicas industriales (Santander et al., 2013), los incendios y fumigaciones (Cevallos, 2015c) junto a la ausencia de sistemas de alcantarillado o de plantas de tratamiento en buen estado (Cevallos, 2011). No obstante, el informe del Censo Neotropical de Aves Acuáticas 2008-2012 ha registrado una biodiversidad notable que convierte al lago en una zona que requiere la implementación de medidas para su protección (Santander et al., 2013) por parte del gobierno descentralizado a cargo, con una prioridad de conservación media-baja, aunque para los humedales alrededor del mismo es alta (Cevallos, 2015c); se debe anotar que el consumo directo del agua debería prohibirse por el nivel de contaminación del agua (Da Ros, 1995). 
En el lago San Pablo los sedimentos son, en su mayoría, orgánicos con un porcentaje de agua de 87,3 \% (Kiersch, Mühleck, \& Gunkel, 2004). Específicamente, es un lago eutrófico débil-moderado que contiene una alcalinidad de $226 \mathrm{mg} / \mathrm{l}$, una conductividad entre 300 y $320 \mathrm{~ms}$ (Terneus, 2014), una concentración alta de fósforo y moderada de nitrógeno (Gunkel, 2000); del mismo modo, se ha calculado que posee niveles elevados de fluoruros, un déficit de oxígeno disuelto y ausencia de nitritos (Tocto, 2013).De forma general, sus tallos se emplean para la elaboración de artesanías (Macía \& Balslev, 2000) y muebles (Cevallos, 2011), con propósitos curativos (De Marco et al., 2011) y en la producción de hojas de papel de pequeño gramaje (De Marco et al., 2011); también existen referencias que demuestran que se utiliza como alimento para los seres humanos (sus rizomas son comestibles (Ramírez \& San Martín, n.d.) y como fertilizante (Banack, Rondón, \& Díaz-Huamanchumo, 2004).

Antiguamente, en los Andes la totora se utilizó para la construcción de casas y puentes (Banack et al., 2004), la tejeduría y como forraje para el ganado (Toensmeier \& Herren, 2016), sin embargo, en las últimas décadas ha adquirido un gran valor económico y cultural para las personas nativas que viven en las regiones en las que crece (Macía \& Balslev, 2000), en especial en Ecuador por la fabricación de balsas, esteras, cestas y abanicos o aventadores (Heiser, 1978).

Se ha comprobado que las plantas acuáticas pueden absorber y acumular de forma fácil los metales pesados (Harguinteguy, Pignata, \& Fernández-Cirelli, 2015), por lo que se han realizado varias investigaciones para determinar el potencial de ciertas especies como bioindicadores y/o fitoremediadores de metales en los medios acuáticos (Peuke \& Rennenberg, 2005), además, el rango de tolerancia de las especies se mide con su respuesta a condiciones hidrológicas diversas (Pratolongo, Kandus, \& Brinson, 2008).

Un estudio corrobora esto al establecer que la presencia de Schoenoplectus californicus en los costados de los cuerpos de agua donde las concentraciones de metales son altas y, por lo tanto, es muy tolerante a los mismos (Arreghini, De Cano, De Lorio, Rendina, \& Bonetto, 2001). Existen diferentes métodos por los cuales las plantas pueden permanecer en ambientes contaminados con metales tóxicos, como la translocación a los tallos, la exudación desde las raíces de sustancias capaces de producir la quelación de metales, la generación de compuestos intracelulares y su acumulación en las vacuolas y otros compartimentos celulares para atenuar los efectos del estrés e incrementar la resistencia interna a los metales (Lasat, Baker, \& Kochian, 1996). En consecuencia, los criterios necesarios para establecer que una planta es hiperacumuladora son valores umbrales mayores a $10 \mathrm{mg} \mathrm{kg}^{-1}\left(10000 \mathrm{\mu g} \mathrm{g}^{-1}\right) \mathrm{de}$ manganeso o zinc, mayor a $1 \mathrm{mg} \mathrm{g}^{-1}\left(1000 \mathrm{\mu g} \mathrm{g}^{-1}\right)$ de arsénico, cobalto, cromo, cobre, níquel, plomo, antimonio, selenio y talio, y mayor de $0,1 \mathrm{mg} \mathrm{g}^{-1}\left(100 \mu \mathrm{g} \mathrm{g}^{-1}\right)$ de cadmio sin atravesar daños fitotóxicos (Boyd, 2004; Rascio \& Navari-Izzo, 2011).

\section{Importancia del problema}

En Ecuador no existen investigaciones que establezcan que Schoenoplectus californicus, que se encuentra en las zonas bentónicas (en los bordes del lago) y que ha sido extraída de la Vertiente Apangora del Lago San 
Pablo-Imbabura, posea una capacidad de hiperacumulación de metales pesados, así como cuáles de ellos se encuentran en un mayor nivel de acumulación.

En el presente, el incremento de la contaminación antropogénica derivada de las actividades industriales y agrícolas ha provocado el deterioro de la calidad del agua y el aumento de la acumulación de sedimentos provocando la disminución del espejo de agua del Lago San Pablo. Por estas razones, es necesario implementar nuevas alternativas de remediación al aprovechar la propiedad que tienen ciertas plantas acuáticas de hiperacumulación de metales pesados; así, se podría descontaminar los suelos y enfocar su uso en técnicas de fitominería para extraer metales pesados con gran potencial económico, como el níquel, $y$, en especial, en aquellos lugares en donde no se puede llevar a cabo su extracción por las técnicas de minería tradicionales por inconvenientes financieros o medioambientales (Nicks\&Chambers, 1995).

\section{METODOLOGÍA}

El área de estudio (como se puede observar en la Figura 3) es la Vertiente Apangora conocida con este nombre por la comunidad indígena que habita en esta zona.

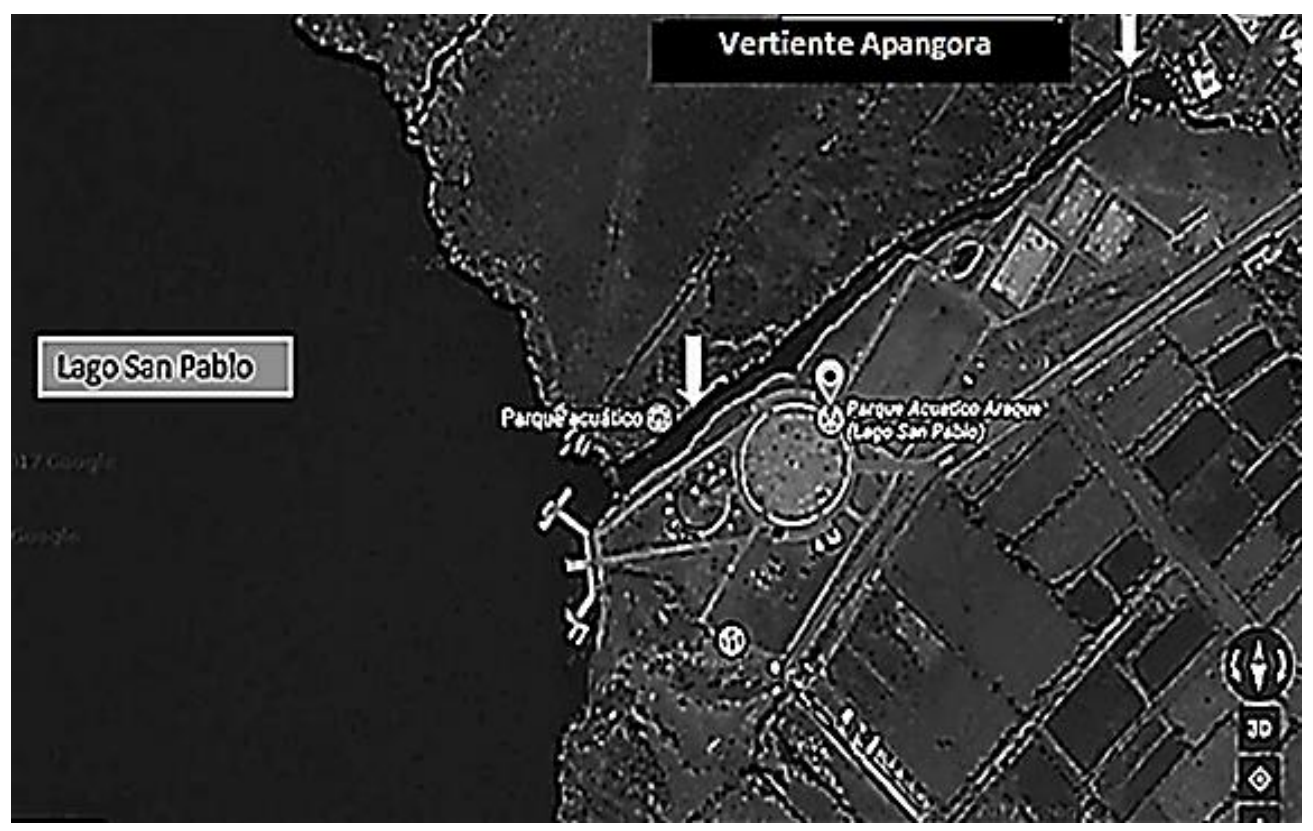

Figura 3 Vertiente Apangora (Imbabura-Ecuador)

Esta vertiente se encuentra ubicada en la parte noroccidental del Lago San Pablo, Imbabura-Ecuador, como se observa en la figura 4: 


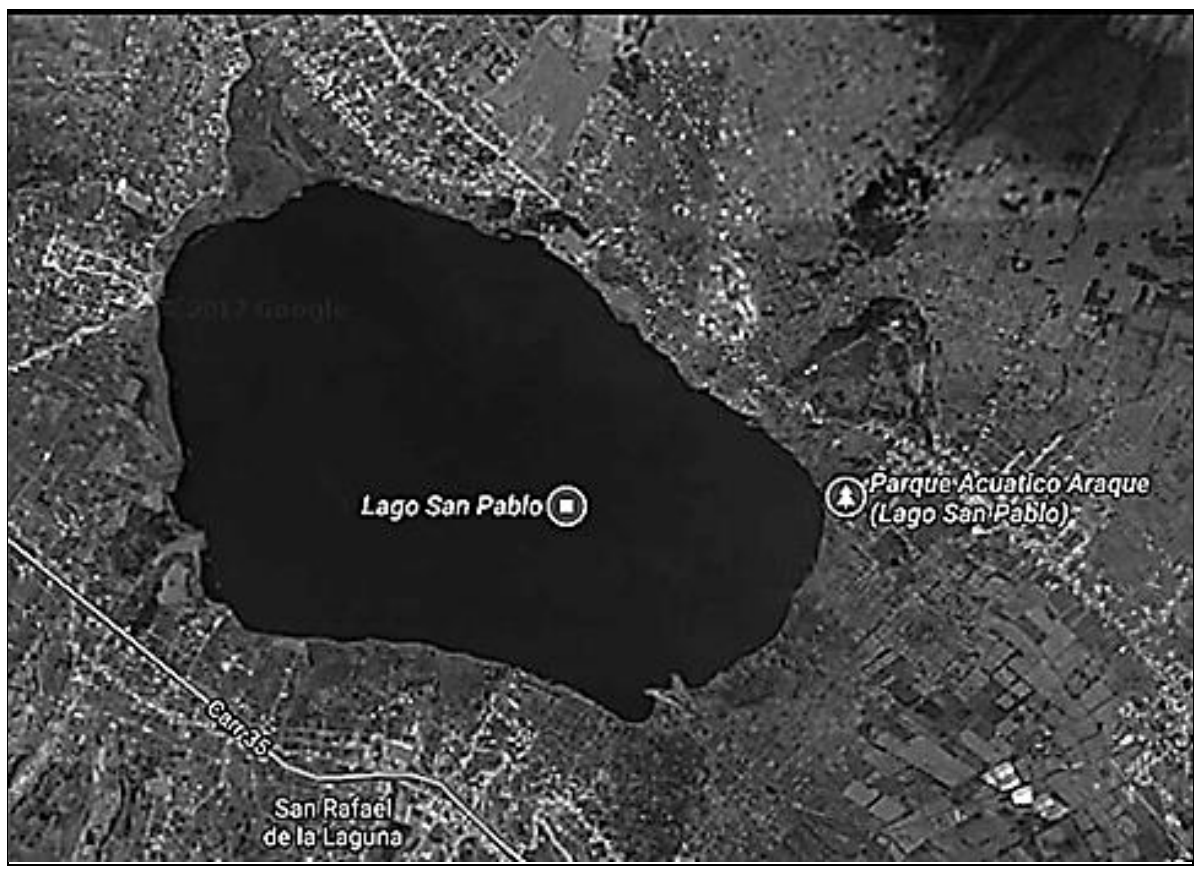

Figura 4 Parque acuático.

Se tomaron muestras de totora con cuatro repeticiones por cada mes de estudio y en total se analizaron doce muestras y se cuantificó la presencia de metales pesados en este macrofito obtenidas en enero, febrero y marzo de 2017.El lugar de muestreo fue en las zonas bentónicas donde desemboca la Vertiente Apangora como afluente al Lago San Pablo como se describe en la Figura 5:

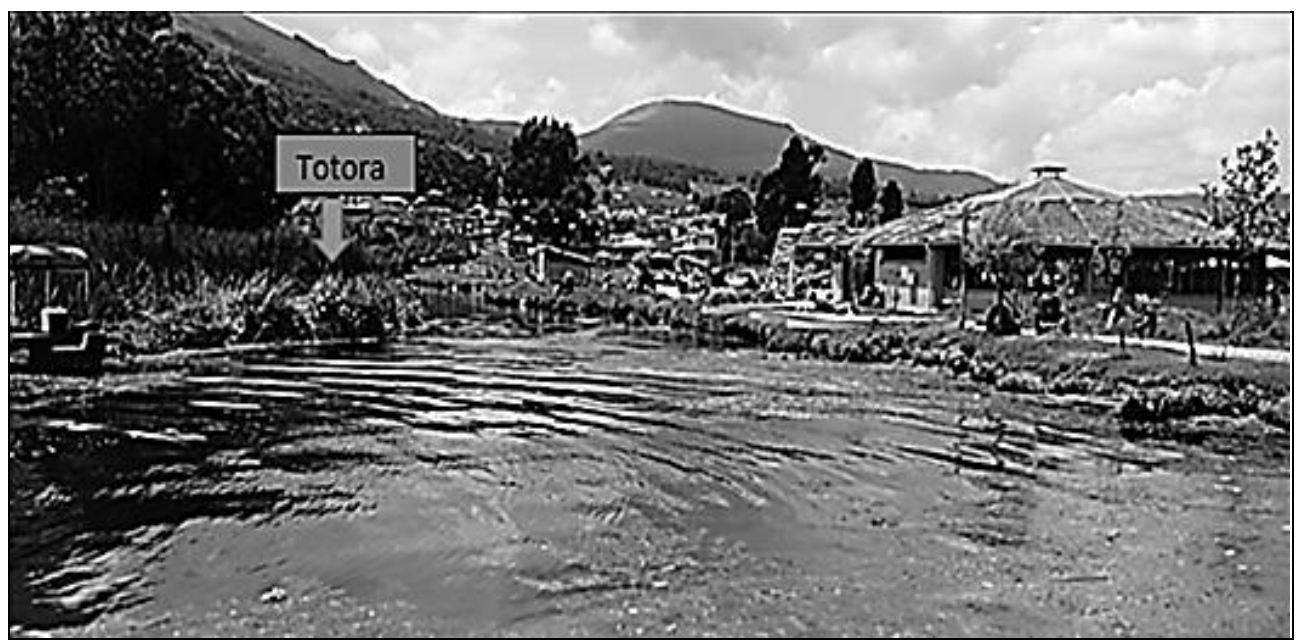

Figura 5 Desembocadura de Vertiente Apangora al Lago San Pablo (Imbabura - Ecuador) 
Se analizó la presencia de metales pesados en las raíces y los tallos de Schoenoplectus californicus mediante el método de absorción atómica en generación de hidruros y horno de grafito y se han cuantificado metales pesados como el mercurio, arsénico, níquel, plomo, cadmio y cromo para determinar su capacidad de hiperacumulación.

Antes de realizar el análisis de absorción atómica mediante el horno de grafito o generador de hidruros, las muestras de raíces y tallos fueron cortadas y secadas a temperatura ambiente durante 7 días. Posteriormente en un vaso de precipitados de $250 \mathrm{ml}$ se llevó a cabo el procedimiento de digestión con $10 \mathrm{~g}$ de muestra y $30 \mathrm{ml}$ de ácido sulfúrico al $98 \%$ (V/V). A continuación se adicionó 100 $\mathrm{ml}$ de agua destilada a la mezcla obtenida, se filtró y trasvasó a un balón de aforo de $100 \mathrm{ml}$ donde se enrasó con agua destilada para determinar en el equipo de absorción atómica las concentraciones de metales pesados.

\section{Método de absorción atómica-generación de hidruros, para la determinación de arsénico y mercurio:}

Las muestras obtenidas se introdujeron en el equipo de absorción atómica-generación de hidruros cuya marca y modelo respectivamente son PERKIN ELMER y MHS 15, que cuenta con el programa AA Winlab Analyst y se seleccionó el sistema de generación hidruros para poder realizar las lecturas de las muestras. Las concentraciones determinadas por este instrumento se expresan en $\mathrm{mg} / \mathrm{L}$, por lo que se ha utilizado la siguiente fórmula para obtenerlas en $\mathrm{mg} / \mathrm{Kg}$ :

$$
\frac{m g}{k g}=\frac{\text { lectura } * F D * \text { aforo muestra } * 1000}{1000 * \text { peso muestra }}
$$

\section{Determinación de cadmio, níquel, cromo y plomo por el método de absorción atómica-por llama directa:}

Para realizar el análisis de las muestras en el equipo de absorción atómica-horno de grafito cuya marca y modelo respectivamente son PERKIN ELMER y HGA, previamente se ha limpiado el atomizador del equipo de absorción atómica con agua que contiene $1,5 \mathrm{ml}$ de $\mathrm{HNO} 3$ conc. /L, el instrumento se ha puesto en autozero, se ha atomizado una muestra preparada y, por último, se ha determinado su concentración. La concentración se lee directamente del instrumento en $\mu \mathrm{g} / \mathrm{L}$ y se han hecho los cálculos correspondientes para obtener estos valores en $\mathrm{mg} / \mathrm{kg}$ con la siguiente fórmula:

$$
\frac{m g}{k g}=\frac{\text { lectura } * F D * \text { aforo muestra } * 1000}{1000 * \text { peso muestra }}
$$




\section{RESULTADOS}

En el análisis realizado mediante absorción atómica para determinar las concentraciones $\left(\mathrm{mg} \mathrm{kg}^{-1}\right)$ de los metales pesados como níquel, arsénico, cadmio, mercurio, plomo y cromo presentes en las raíces y el tallo de Schoenoplectus californicus durante el primer semestre de 2017, se obtuvieron los resultados detallados a continuación en la Figura 6:

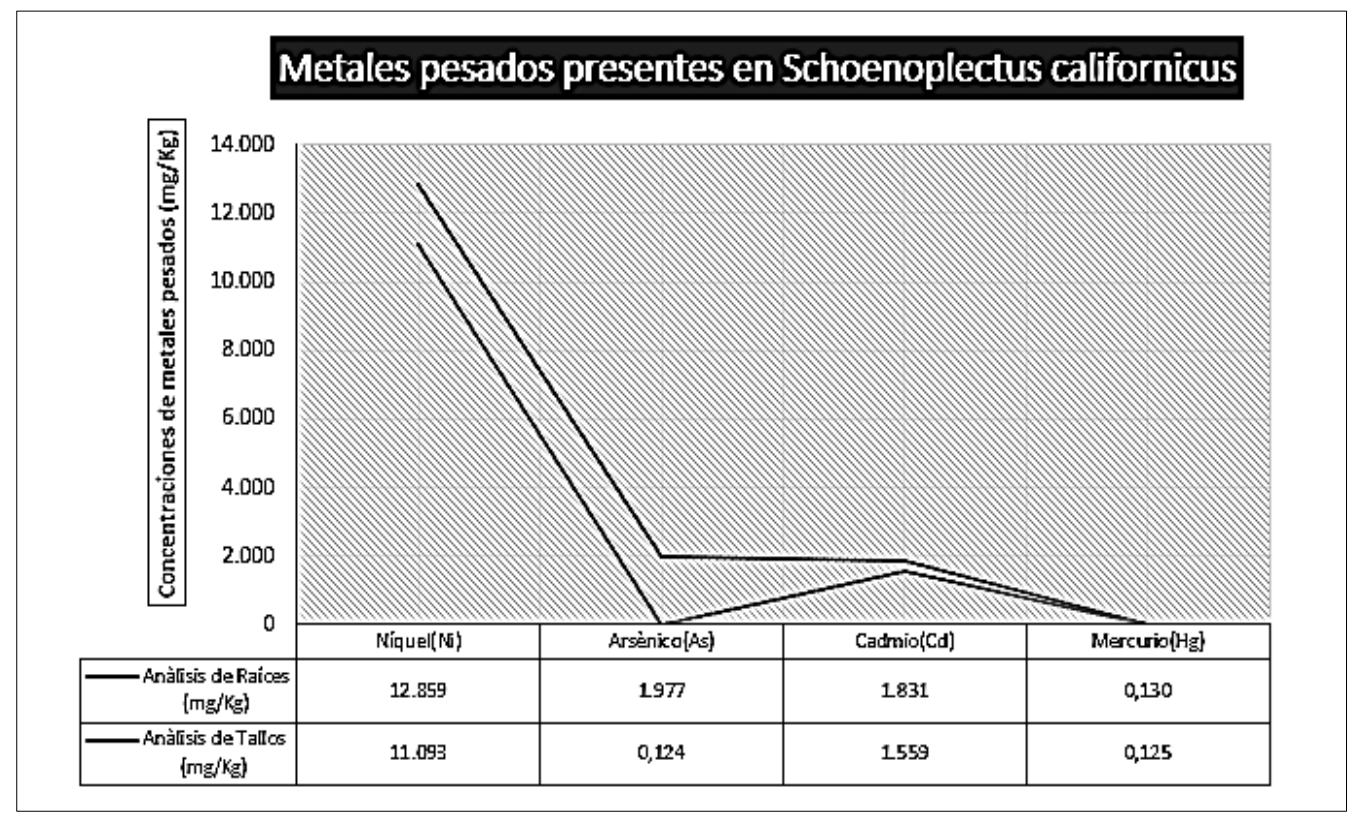

Figura 6 Metales pesados presentes en Schoenoplectus californicus (Primer Trimestre, 2017).

Se puede observar que en las raíces (Ver Figura 6) se ha encontrado la presencia de los siguientes metales: el níquel se presenta con una concentración promedio de 12,859 $\mathrm{mg} \mathrm{kg}^{-1}$, el arsénico en 1,977 $\mathrm{mg} \mathrm{kg}^{-1}$, el cadmio en 1,831 mg $\mathrm{kg}^{-1} \mathrm{y}$ el mercurio en $0,130 \mathrm{mg} \mathrm{kg}^{-1}$.Mientras que, en los tallos (Ver Figura 6), se ha observado que hay concentraciones menores de los siguientes metales: se ha cuantificado que el níquel se encuentra presente en una cantidad de 11,093 mg $\mathrm{kg}^{-1}$, el cadmio en $1,559 \mathrm{mg} \mathrm{kg}^{-1}$, el arsénico en $0,124 \mathrm{mg} \mathrm{kg}^{-1}$ y el mercurio sus concentraciones en $0,125 \mathrm{mg} \mathrm{Kg}^{-1}$. Tanto en el análisis de raíces como de tallos no se ha encontrado concentraciones de plomo y cromo.

Para establecer que metales pesados superan el umbral de hiperacumlación se ha utilizado el análisis estadístico t-student como se detalla en la siguiente tabla 1. 
Tabla 1 Análisis estadístico t de Student

\begin{tabular}{|l|c|c|c|}
\hline \multicolumn{4}{|c|}{ PRUEBA t DE STUDENT } \\
\hline Estadistico $t=2,892$ & $>$ & Valor critico de $t$ (dos colas $)=2,447$ & $\mathrm{P}(\mathrm{T}<=\mathrm{t})$ dos colas $=0,028$ \\
\hline
\end{tabular}

En el análisis estadístico de las concentraciones promedio de metales pesados presentes en las raíces se obtuvo los siguientes valores de media, desviación estándar y coeficiente de variación como se detalla a continuación (Ver tabla 2).

Tabla 2 .Análisis estadístico de las concentraciones promedio de raíces y tallos de Schoenoplectus californcus

\begin{tabular}{|l|c|c|}
\hline \multirow{2}{*}{$\begin{array}{c}\text { Análisis de las } \\
\text { raíces y tallos de la } \\
\text { planta acuática }\end{array}$} & Medía y desviación estándar & Coeficiente de variación \\
\cline { 2 - 3 } & & $\mathrm{CV}=1,30$ \\
\hline Análisis de raíces & $4,200 \pm 5,834 \mathrm{mg} \mathrm{Kg}-1$ & $\mathrm{CV}=1,64$ \\
\hline Análisis de tallos & $3,224 \pm 5,288 \mathrm{mg} \mathrm{Kg}-1$ & \multicolumn{2}{|c|}{ MEDIDAS ESTADíSTICAS } \\
\hline
\end{tabular}

Para determinar si existen diferencias entre los valores obtenidos de las concentraciones de níquel, cadmio, arsénico y mercurio presentes en raíces y tallos se utilizó la prueba estadística de análisis de varianza ANOVA como se detalla a continuación:

Tabla 3 Análisis de varianza de las concentraciones promedio de metales presados presentes en tallos y raíces

\begin{tabular}{|l|l|c|}
\hline \multicolumn{3}{|c|}{ ANÁLISIS DE VARIANZA (ANOVA) } \\
\hline Probabilidad obtenida $=0,032$ & $<$ & $\alpha=0.05$ \\
\hline
\end{tabular}

\section{DISCUSIÓN}

El periodo de análisis de los metales pesados presentes en los tallos y las raíces de este macrofito para determinar las concentraciones de los metales de níquel, arsénico, mercurio, cadmio, cromo y plomo corresponden al primer trimestre del año 2017.

Para establecer el umbral de hiperacumulación de Schoenoplectus californicus se han tomado como referencia los criterios de (Boyd, 2004; Rascio \& Navari-Izzo, 2011) y Muddarisna et. al, 2013 que establecen que una planta es hiperacumuladora cuando tiene valores umbrales mayores a $10 \mathrm{mg} \mathrm{kg}^{-1}$ de mercurio, mayores a $1 \mathrm{mg} \mathrm{kg}^{-1}$ de arsénico, cobalto, cromo, cobre, níquel, plomo y mayores de $0,1 \mathrm{mg} \mathrm{kg}^{-1}$ de cadmio antes de que atraviesen daños fitotóxicos. 
Dentro de las concentraciones promedio de los metales pesados obtenidos en las en las raíces, se observa que las del níquel y arsénico son de $12.859 \mathrm{mg} \mathrm{kg}^{-1}$ y $1.977 \mathrm{mg} \mathrm{kg}^{-1}$, respectivamente como se detalla en la Figura 7 , por lo tanto, estos valores son superiores al umbral de hiperacumulación (mayores a $1 \mathrm{mg} \mathrm{kg}^{-1}$ ). Por otra parte, al analizar las concentraciones promedio de estos metales pesados en los tallos, se ha observado que el níquel y arsénico se presentan en concentraciones promedio de $11.093 \mathrm{mg} \mathrm{kg}^{-1}$ y $0.124 \mathrm{mg} \mathrm{kg}^{-1}$ (Ver Figura 7), respectivamente. Así, se puede establecer que, solamente el níquel ha superado el umbral de hiperacumulación de una planta hiperacumuladora (mayor a $1 \mathrm{mg} \mathrm{kg}^{-1}$ ).

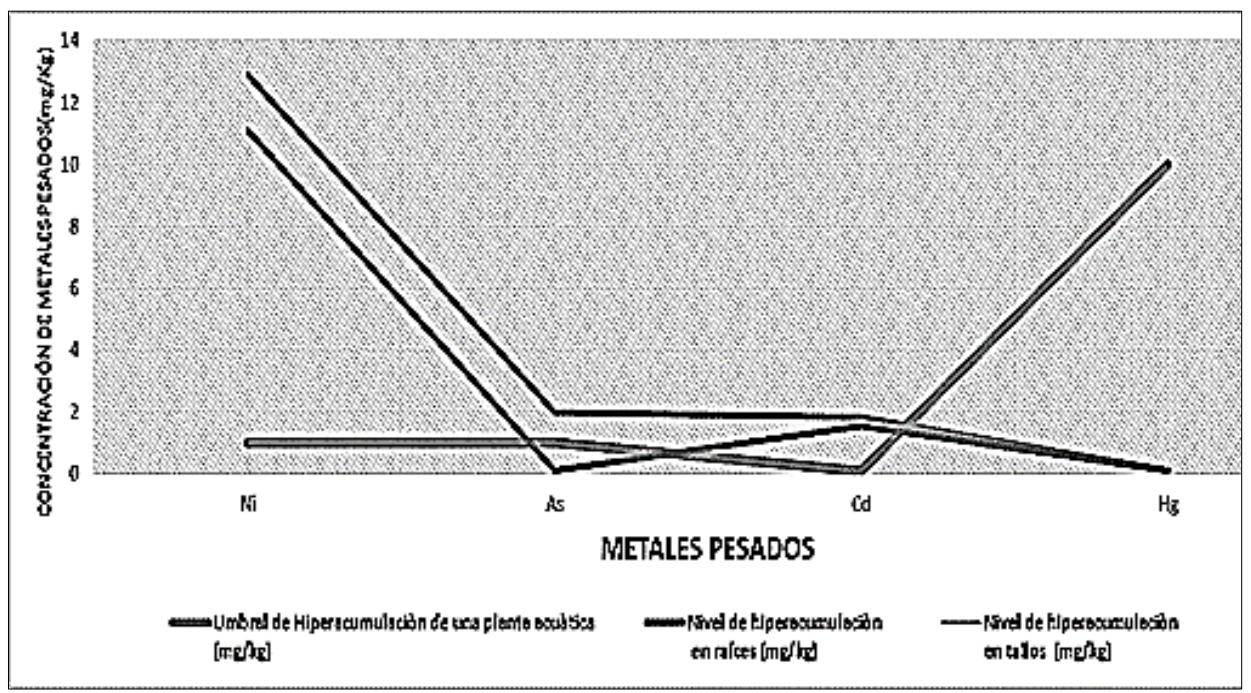

Figura 7 Representación de los niveles de hiperacumulación obtenidos en Schoenoplectus californicus (Primer Trimestre, 2017).

Las concentraciones promedio de cadmio en las raíces y los tallos son $1.831 \mathrm{mg} \mathrm{Kg}^{-1}$ y $1.559 \mathrm{mg} \mathrm{Kg}^{-1}$ (Ver Figura 7), respectivamente, y al compararlas con el umbral de hiperacumulación establecido para este metal de $0,1 \mathrm{mg} \mathrm{Kg}^{-1}$, se aprecia que estos valores lo superan. La cuantificación de los niveles de mercurio en las raíces y los tallos se ha determinado en valores de $0.13 \mathrm{mg} \mathrm{Kg}^{-1}$ y $0.12 \mathrm{mg}$ $\mathrm{Kg}^{-1}$ (Ver Figura 7) y, al compararlos con el umbral de hiperacumulación establecido para este metal de $10 \mathrm{mg} \mathrm{Kg}^{-1}$, se puede decir que este metal no supera el umbral de hiperacumulación.

Al obtener el valor estadístico t mayor al valor absoluto crítico de $t$ dos colas (Ver Tabla 1), se establece que se acepta la hipótesis alternativa con una probabilidad de 0,028 y, por lo tanto, existe diferencias estadísticamente significativas entre los valores del umbral de hiperacumulación y los valores obtenidos de hiperacumulación de la Schoenoplectus californicus. Además se constata que los niveles de hiperacumulación de este macrofito son superiores para el níquel y el cadmio en los tallos y raíces, mientras que la concentración de arsénico solamente es superior en las raíces. 
Con los valores de las medias y sus desviaciones estándar obtenidos de las concentraciones promedios de las raíces y de sus tallos se observa que existe variabilidad entre ellos (Ver Tabla 2).Además se ha constatado que existe diferencia de valores obtenidos entre los coeficientes de variación (Ver Tabla 2), esto indica que existe dispersión entre ellos y, en consecuencia, son considerados como heterogéneos, es decir, las concentraciones promedio de metales de níquel, arsénico, cadmio y mercurio tanto de las raíces como de los tallos no son consideradas como no datos homogéneos, y por tanto existen metales pesados que se encuentran unos en mayor proporción que otros.

La probabilidad obtenida del ANOVA es de $p=0,032$ (Ver tabla 3) y, al compararla con el nivel de significancia, se puede determinar que el valor de $p$ obtenido es menor que el nivel de significancia, con un nivel de confianza del $95 \%$. Por tanto, aceptando la hipótesis alternativa que considera que las medias aritméticas de las concentraciones de metales pesados son diferentes en las raíces que en los tallos de Schoenoplectus californicus, se ha corroborado que existen mayores concentraciones de níquel, arsénico, cadmio y mercurio presentes en las que raíces que los tallos.

\section{COMENTARIOS Y CONCLUSIONES}

Se concluye que, para el primer trimestre 2017 de análisis de metales pesados, existen mayores concentraciones de estos en las raíces que en los tallos de Schoenoplectus californicus. Las razones se deben a que en los tallos de la totora se desarrollan mecanismos de defensa para evitar la absorción de metales no esenciales y, por esta razón, los metales pesados se mantienen en las raíces para que solo en situaciones de altas concentraciones migren hacia los rizomas (Arreghini et al., 2001)

Se ha encontrado que en las raíces de este macrofito se encuentran en mayor proporción los siguientes metales pesados en orden de mayor a menor: níquel, arsénico, cadmio y mercurio $(\mathrm{Ni}>\mathrm{As}>\mathrm{Cd}>\mathrm{Hg}$ ). Por otro lado, en los tallos se encuentran en mayor proporción el níquel, cadmio, mercurio y arsénico $(\mathrm{Ni}>\mathrm{Cd}>\mathrm{Hg}>\mathrm{As})$.

Además, mediante el método de absorción atómica en horno de grafito con una sensibilidad muy alta para la cuantificación de metales traza, no se ha encontrado valores de cromo y plomo presentes en este macrofito en el primer trimestre 2017.

Por otra parte, se ha detectado que las concentraciones de níquel, cadmio y arsénico cuantificadas en Schoenoplectus californicus presentan valores superiores al umbral de hiperacumulacion tomando como referencia los criterios de (Boyd, 2004; Rascio \& Navari-Izzo, 2011) y Muddarisna et. al, 2013 . Por lo tanto, esta característica podría representar una oportunidad para generar los medios que permitan su aplicación en la descontaminación de suelos y fitominería.

En próximos estudios, se podrá esclarecer si la concentración de estos metales se debe a una tendencia natural hacia una mayor absorción de estos particularmente o si, por el contrario, existe una concentración significativamente 
más elevada en el periodo analizado (primer trimestre de 2017) debido al influjo de fuentes externas de contaminación.

\section{REFERENCIAS}

1. Arreghini, S., De Cano, L., De Lorio, A., Rendina, A., \& Bonetto, C. (2001). Effects of zinc on the growth of bulrush (Schoenoplectus californicus) and its distribution between different sediment fractions. Bulletin of Environmental Contamination and Toxicology, 67(2), 264-270. http://doi.org/10.1007/s001280119

2. Banack, S. A., Rondón, X., \& Díaz-Huamanchumo, W. (2004). Indigenous Cultivation and Conservation of Totora (Schoenoplectus californicus, Cyperaceae) in Peru. EBSCO, 58(1), 11-20. Retrieved from http://web.a.ebscohost.com/abstract?direct=true\&profile=ehost\&scope=site\&authty $\mathrm{pe}=$ crawler\&jrnl $=00130001 \& \mathrm{AN}=13853257 \& \mathrm{~h}=2 \mathrm{Tc0} 1 \mathrm{a} 4 \mathrm{~K} 2 \mathrm{qePZuYhicZU2a6DA4Cl}$ G45SR2HfpgekuiZtBkCXgXfJ3JibHQhsdsED\%2BQ4E5ejnJjGgCLCAlHdDcA\%3D $\% 3 \mathrm{D} \& \mathrm{crl}=\mathrm{c} \&$ resultNs=AdminWebAuth\&resultLocal $=\mathrm{Er}$

3. Boyd, R. S. (2004). Ecology of metal hyperaccumulation. New Phytologist, 162(3), 563-567. http://doi.org/10.1111/j.1469-8137.2004.01079.x

4. Casallas, J. E., \& Gunkel, G. (2001). Algunos aspectos limnológicos de un lago altoandino: el lago San Pablo, Ecuador. Asociación Española de Limnología, 215-232. Retrieved from http://www.limnetica.com/Limnetica/Limne20/L20b215_Limnologia_lago_S an_Pablo_Ecuador.pdf

5. Cevallos, M. P. (2011). Plan de desarrollo y ordenamiento territorial de la parroquia San Rafael de la Laguna. Otavalo. Retrieved from http://www.sanrafaeldelalaguna.gob.ec/pdot.pdf

6. Cevallos, M. P. (2015a). Actualización del plan de desarrollo y ordenamiento territorial de la parroquia Eugenio Espejo 2015 - 2019. Otavalo. Retrieved from

http://app.sni.gob.ec/snlink/sni/PORTAL_SNI/data_sigad_plus/sigadplusdo cumentofinal/1060013670001_PDOT EUGENIO ESPEJO_30-102015_12-46-42.pdf

7. Cevallos, M. P. (2015b). Plan de desarrollo y ordenamiento territorial de la parroquia Gonzalez Suarez 2015 - 2019. Otavalo. Retrieved from http://app.sni.gob.ec/sni-

link/sni/PORTAL_SNI/data_sigad_plus/sigadplusdocumentofinal/10600153 70001_PDOT GŌNZALEZ SUEREZ_30-10-2015_11-25-27.pdf

8. Cevallos, M. P. (2015c). Plan de desarrollo y ordenamiento territorial de la parroquia San Pablo. San Pablo. Retrieved from http://app.sni.gob.ec/snilink/sni/PORTAL_SNI/data_sigad_plus/sigadplusdocumentofinal/10600147 20001_PDOT SAN PABLO_27-10-2015_11-33-45.pdf 
9. Chester, E., Wofford, E., Estes, D., \& Bailey, C. (2009). A fifth checklist of Tennessee vascular plants (1st Ed.). Texas: BRIT Press. Retrieved from https://books.google.es/books?id=xh-

NE2dRdNsC\&pg=PA15\&dq=Schoenoplectus+californicus+or+Scirpus\&hl= es\&sa $=$ X\&ved=0ahUKEwiN8OL4-

d7SAhUJ7iYKHRTPBpUQ6AEIMTAD\#v=onepage\&q=Schoenoplectus californicus or Scirpus\&f=false

10. Da Ros, G. (1995). La contaminación de aguas en Ecuador: una aproximación económica. Instituto de Investigaciones Económicas, P.U.C.E. Retrieved from https://books.google.es/books?id=rPQrAHRxzyYC\&pg=PA73\&lpg=PA73\& $\mathrm{dq}=$ contaminación+lago+san+pablo\&source=bl\&ots=PORAPv4oOF\&sig= GkJNyyo3tvCTh9Jox08sSnBLdMM\&hl=es\&sa=X\&ved=0ahUKEwi1h4aRk enSAhWG6yYKHXasD7AQ6AEIPDAI\#v=onepage\&q=contaminación lagos

11. De Marco, S., Vega, L., \& Bellagamba, P. (2011). La reserva natural del Puerto Mar del Plata, un oasis urbano de vida silvestre. ( $1^{\text {a }}$ Ed.). Mar del Plata: Universidad FASTA. Retrieved from https://books.google.es/books?id=4HbGN4ssGeAC\&pg=PA132\&dq=Scho enoplectus+californicus+junco\&hl=es\&sa $=X \& v e d=0 a h U K E w i w 7 c z r q 9 z S A h$ XSdSYKHRI-CacQ6AEIHjAB\#v=onepage\&q=Schoenoplectus californicus junco\&f$=f a l s e$

12. Gunkel, G. (2000). Limnology of an equatorial high mountain lake in Ecuador, Lago San Pablo. Limnologica - Ecology and Management of Inland Waters, 30(2), 113-120. http://doi.org/10.1016/S00759511(00)80005-5

13. Harguinteguy, C. A., Pignata, M. L., \& Fernández-Cirelli, A. (2015). Nickel, lead and zinc accumulation and performance in relation to their use in phytoremediation of macrophytes Myriophyllum aquaticum and Egeria densa. Ecological Engineering, 82, 512-516. http://doi.org/10.1016/j.ecoleng.2015.05.039

14. Heiser, C. (1978). The Totora (Scirpus Californicus) in Ecuador and Peru. Economic Botany, 32(3), 222-236. http://doi.org/10.1007/BF02864698

15. Jurado, P., Jaramillo, M. G., \& Reyes, W. (2015). Actualización del plan de desarrollo y ordenamiento territorial de la provincia de Imbabura 20152035. Imbabura. Retrieved from http://app.sni.gob.ec/snilink/sni/PORTAL_SNI/data_sigad_plus/sigadplusdocumentofinal/10600001 80001_PDOT IMBABURA 2015-2035_SIGAD_15-08-2015_22-50-42.pdf

16. Kiersch, B., Mühleck, R., \& Gunkel, G. (2004). Las macrófitas de algunos lagos alto-andinos del Ecuador y su bajo potencial como bioindicadores de eutrofización. Rev. Biol. Trop. (Int. J. Trop. Biol, 52(4), 829-837. Retrieved from http://www.ots.ac.cr/rbt/attachments/volumes/vol52-4/01KIERSCH mac.pd 
17. Lasat, M. M., Baker, A. J. M., \& Kochian, L. V. (1996). Physiological Characterization of Root Zn2+ Absorption and Translocation to Shoots in $\mathrm{Zn}$ Hyperaccumulator and Nonaccumulator Species of Thlaspi. Plant Physiol, 11(2), 171-5. Retrieved from https://www.ncbi.nlm.nih.gov/pmc/articles/PMC158105/pdf/1121715.pdf

18. López, Z. (2012). Zonificación ecológica del lago san pablo en función de los patrones de distribución espacial y temporal de la avifauna acuática. Otavalo. Retrieved from http://repositorio.utn.edu.ec/bitstream/123456789/2091/1/03 RNR 156 ARTíCULO.pdf

19. Ninaquispe-Romero, L., Weeks, S., \& Huelman, P. (2012). Totora: A Sustainable Insulation Material for the Andean Parts of Peru. In 28th Conference, Opportunities, Limits \& Needs Towards an environmentally responsible architecture (p. 7). Lima: PLEA2012. Retrieved from http://plea-arch.org/ARCHIVE/2012/files/T02-20120130-0067.pdf

20. Macía, M. J., \& Balslev, H. (2000). Use and management of Totora (Schoenoplectus Californicus, Cyperaceae) in Ecuador. Economic Botany, 54(1), 82-89. http://doi.org/10.1007/BF02866602

21. Peuke, A. D., \& Rennenberg, H. (2005). Phytoremediation. EMBO Reports, 6(6), 497-501. http://doi.org/10.1038/sj.embor.7400445

22. Pratolongo, P., Kandus, P., \& Brinson, M. (2008). Net Aboveground Primary Production And Biomass Dynamics Of Schoenoplectus Californicus (Cyperaceae) Marshes Growing Under Different Hydrological Conditions. SCIELO, 46(2), 258-269. Retrieved from http://www.scielo.org.ar/scielo.php?script=sci_arttext\&pid=S001167932008000200006

23. Rascio, N., \& Navari-Izzo, F. (2011). Heavy metal hyperaccumulating plants: How and why do they do it? And what makes them so interesting? Plant Science, 180(2), 169-181. http://doi.org/10.1016/j.plantsci.2010.08.016

24. Ramírez, C., \& San Martín, C. (n.d.). Flora acuática. Santiago de Chile. Retrieved from http://www.mma.gob.cl/librobiodiversidad/1308/articles45206_recurso_4.pdf

25. Richardson, A., \& King, K. (2010). Plants of deep south Texas: a field guide to the woody \&amp; flowering species ( $1^{\text {a }} \mathrm{Ed}$.). Texas: Texas A \& M University Press. Retrieved from https://books.google.es/books?id=ve-fHYDy8wC\&pg=PA38\&dq=Schoenoplectus+californicus\&hl=es\&sa $=X \& v e d=0 a$ hUKEwiF_4eEq9zSAhXCVyYKHUtrCzwQ6AEIYjAJ\#v=onepage\&q=Schoe noplectus californicus\& $f=$ false

26. Santander, T., Ágreda, A., \& Adriana, L. (2013). Censo neotropical de aves acuáticas Ecuador 2008 - 2012. Quito. Retrieved from 
http://lac.wetlands.org/Portals/4/CNAA 2008/2012/CNAA_info2008-2012 EC fin.pdf

27. Sistema Nacional de Información. (2011). Sistema ambiental. Imbabura. Retrieved from http://app.sni.gob.ec/snilink/sni/PDOT/ZONA1/NIVEL_DEL_PDT_PROVINCIAL/INFORMACION GAD/01 GPI_GOBIERN̄O PROVINCIAL IMBABURA/cap2_3_Diag_SistAmbienta.pdf

28. Terneus, E. (2014). Vegetación acuática y estado trófico de las lagunas andinas de San Pablo y Yahuarcocha, provincia de Imbabura, Ecuador. REMCB, 35, 121-131. Retrieved from http://uide.edu.ec/media/1347/9vegetacion-acuatica-estado-trofico-lagunas-andinas-san-pabloyahuarcocha-imbabura.pdf

29. Tocto, A. (2013). Evaluación de la calidad del agua en la parroquia San Pablo del Lago, cantón Otavalo, provincia de Imbabura, utilizando un cromatógrafo de intercambio iónico con supresión química, previamente validado por el método APHA4110. Escuela Politécnica del Ejército. Retrieved from http://repositorio.espe.edu.ec/bitstream/21000/7391/1/ACB-ESPE-047516.pdf

30. Toensmeier, E., \& Herren, H. R. (2016). The carbon farming solution: a global toolkit of perennial crops and regenerative agriculture practices for climate change mitigation and food security (1st Ed.). Vermont: Chelsea Green Publishing. Retrieved from https://books.google.es/books?id=zsh2CwAAQBAJ\&pg=PA253\&dq=Schoe noplectus+cal

31. USDA. (n.d.). Plants Profile for Schoenoplectus californicus (California bulrush). Retrieved March 18, 2017, from https://plants.usda.gov/core/profile?symbol=scca11 